\title{
The Effects of Solvent, Molecular Weight, and Concentration on the Fold Length of Solution-Grown Polymer Single Crystals
}

\author{
Fumiyuki Hamada, Takashi Korenaga, and Akio Nakajima \\ Department of Polymer Chemistry, Kyoto University, \\ Sakyo-ku, Kyoto, Japan.
}

(Received December 22, 1975)

\begin{abstract}
The effects of the kind of solvents, the molecular weight of the polymer, and the concentration of solute in solution on the fold length of polymer single crystals formed from solution were investigated. It was found that these effects on the fold length of polymer single crystals were explained very well from kinetic theory but not from equilibrium theory. It was concluded that the chain folded polymer crystallization, therefore, normally proceeds by means of the kinetic mechanism of secondary nucleation.
\end{abstract}

KEY WORDS Single Crystal / Fold Length / Degree of Supercooling / Solvent Effect / Kinetic Theory /

A theory of chain folding with any claim to validity needs to explain why chains should fold, why the fold length is uniform within the limits observed, and why the fold length varies with the crystallization temperature in the manner observed. In addition, it has to be at least consistent with the fold length increase on annealing. Till now, two theoretical treatments have been developed which can be compared with experimental results. One, the equilibrium theory developed by Peterlin, Fischer, and Reinhold, ${ }^{1}$ suggests that the fold length is determined thermodynamically, and corresponding to a minimum in the free energy density of the crystals at the crystallization temperature. The other, the kinetic theory suggested independently by Price, ${ }^{2}$ Lauritzen and Hoffman, ${ }^{3}$ and Frank and Tosi ${ }^{4}$ on the basis of a kinetic approach, was subsequently developed and applied to crystallization from both solution and melt. According to the kinetic theory, the observed folded chain crystals correspond to the structure with maximum probability. Both theories succeeded in accounting for the observed temperature dependence of fold length.

We determined previously the fold length of single crystals of polyethylene, poly(4-methyl1 -pentene), and poly(oxymethylene) grown from various solvents at different temperatures and found that the fold length of the single crystal formed at the same temperature is dependent of the kind of solvent. ${ }^{5-7}$ In this paper we investigate the effects of the solvent, the molecular weight, and the concentration of solute on the fold length of polymer single crystal from solution and discuss by using these results which theory is valid, the equilibrium theory or the kinetic theory.

\section{EXPERIMENTAL}

\section{Polymer Samples}

For the polyethylene samples, molecular weight fractions of linear polyethylene (Sholex 6050) were used. The fractionation was carried out by using a large scale fractionation column which was designed by Nakajima, et al. ${ }^{8}$ For poly(4-methyl-1-pentene), the polymer sample polymerized by use of Ziegler catalysis was extracted for $48 \mathrm{hr}$ with boiling xylene and the residue was used. For poly(oxymethylene), Derlin 500 was extracted for $24 \mathrm{hr}$ at $110^{\circ} \mathrm{C}$ with a mixture of phenol and ethyl cellosolve $(95: 5)$ and the residue was used. The molecular weights of the polymer samples used in the discussion of the solvent effect were $4.2 \times 10^{4}$ in polyethylene, $10^{6}$ in poly(4-methyl-1-pentene), and $1.8 \times 10^{5}$ in poly(oxymethylene). 


\section{F. Hamada, T. Korenaga, and A. Nakajima}

\section{Preparation of Single Crystals}

Single crystals of polyethylene, poly(4-methyl1-pentene), and poly(oxymethylene) were prepared at various temperatures from $0.1 \%$ solutions in various solvents, according to the method described in a previous paper. $^{5-7}$ Polyethylene single crystals were prepared from $1 \%$ solution in xylene by the same method, but in the preparation of polyethylene single crystals from $5 \%$ solution in xylene, the single crystals tend to crystallize at a higher temperature than that desired. To avoid this trouble, crystallization was carried out by putting the concentrated solution in a flat bag made of aluminium foil and immersing the bag into a thermostated bath at a given temperature.

\section{Measurement of Fold Length of Single Crystals}

The fold length was calculated from the photograph of the small-angle X-ray diffraction pattern taken by a Rigaku-Denki small-angle X-ray camera with pinhole collimation.

\section{Determination of Dissolution Temperature}

The dissolution temperature $T_{\mathrm{s}}$ used in the discussion of the solvent effect was determined as follows. For polyethylene, ${ }^{5} T_{\mathrm{s}}$ was determined using an extended chain crystal of polymethylene crystallized under high pressure, ${ }^{9}$ which was kindly supplied by Dr. B. Wunderlich. For poly(4-methyl-1-pentener), ${ }^{6} T_{\mathrm{s}}$ was determined by use of a polymer sample sufficiently crystallized at a temperature near the melting temperature. For poly(oxymethylene), ${ }^{7} T_{\mathrm{s}}$ was determined by use of an extended chain crystal of poly(oxymethylene) obtained by radiation-induced solid state polymerization of trioxane. ${ }^{10}$

\section{RESULTS AND DISCUSSION}

\section{Effect of Solvent}

According to the kinetic theory by Lauritzen and Hoffman, ${ }^{3}$ the lamellar thickness of polymer single crystals grown at $T_{\mathrm{c}}$ is given by the following equation:

$$
\bar{l}=\delta 1+2 \sigma_{\mathrm{e}} / \Delta f
$$

where $\bar{l}$ is the average lamellar thickness of the crystals, $\delta l$ an "extra" size term, $\sigma_{\mathrm{e}}$ the surface free energy of the fold-containing surface, and $\Delta f$ the free energy difference between the super- cooled liquid and the crystal phases. $\delta l$ is expressed in various ways by different theories. ${ }^{3,4,11}$ All these expressions have the common feature that they yield a $\delta l$ value of $5-15 \AA$ for a not too large degree of supercooling. The term $\Delta f$ can be approximated by the expression

$$
\Delta f=\frac{\Delta h_{\mathrm{f}}\left(T_{\mathrm{s}}-T_{\mathrm{c}}\right)}{T_{\mathrm{s}}}
$$

where $\Delta h_{\mathrm{f}}\left(\mathrm{erg} / \mathrm{cm}^{3}\right)$ is the heat of fusion per unit volume of a perfect crystal at the melting temperature, $T_{\mathrm{c}}$ the crystallization temperature, and $T_{\mathrm{s}}$ is the dissolution temperature, which may be defined as that temperature at which the polymer solution is in equilibrium with the extended crystal of that polymer. ${ }^{12}$ Combining eq 1 and 2 , we obtain the following equation:

$$
\bar{l}=\delta 1+\frac{2 \sigma_{\mathrm{e}} T_{\mathrm{s}}}{\Delta h_{\mathrm{f}}\left(T_{\mathrm{s}}-T_{\mathrm{c}}\right)}
$$

The value of $\sigma_{\ominus}$ of a polymer single crystal from solution was estimated from eq 3 in previous papers. $^{5-7} \quad \sigma_{\mathrm{e}}$ of polyethylene single crystal $^{5,7}$ somewhat varys with solvents, while $\sigma_{\mathrm{e}}$ of poly(4methyl-1-pentene) ${ }^{6}$ and poly(oxymethylene) single crystal are independent of solvents. ${ }^{*}$ It may be concluded, therefore, that $\sigma_{\mathrm{e}}$ of a polymer single crystal does not differ significantly unless solvents with very different thermodynamic properties are used. If $\sigma_{e}$ is independent of solvents, from eq 3 the lamellar thickness should be inversely proportional to the degree of supercooling, $T_{\mathrm{s}}-T_{\mathrm{c}}$. Accordingly, it should strongly depend on the solvent. As the equilibrium theories are essentially based on lattice dynamics, the fold length should be essentially independent of the solvent and be a function of actual temperature. The solvent dependence of the fold length of a solution-grown single crystal was first studied by Fischer, et al. ${ }^{13}$ who determined the fold lengths of polyethylene single crystals grown from various solvents at different temperatures and reported that the fold length is a function of the crystallization temperature irrespective of the kind of solvents. Kawai and

* In a previous paper, ${ }^{6} \sigma_{\mathrm{e}}$ of poly(4-methyl-1-pentene) was calculated erroneously. The correct values of $\sigma_{e}$ are $28,30,26$, and $31\left(\mathrm{erg} / \mathrm{cm}^{2}\right)$ for decalin, $p$-xylene, tetralin, and $n$-hexadecane, respectively. 
Keller $^{14}$ found that the fold lengths of single crystals of polyethylene from xylene and octane are different even when they are crystallized at the same crystallization temperature.

The plots of the fold length $\bar{l}$ against the crystallization temperature $T_{\mathrm{c}}$ of polyethylene, poly(4-methyl-1-pentene), and poly(oxymethylene) are reproduced in Figures 1, 2, and 3, respectively, from previous papers. ${ }^{5-7}$ As is obvious from the figures, the fold length is dependent on the kind of solvents. Furthermore, in Figure 4 , the fold length $\bar{l}$ is plotted against the reciprocal of the degree of supercooling for polyethylene, poly(4-methyl-1-pentene), and poly(oxymethylene) single crystal. The values of the dissolution temperatures of the polymer single crystals used

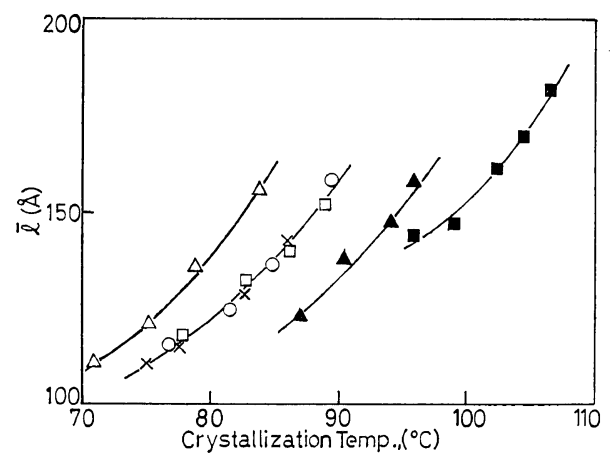

Figure 1. Fold length against crystallization temperature for polyethylene single crystals grown from dilute solutions: 5 (ㅁ) $n$-hexadecane; (A) $n$ octane; ( $\bigcirc$ ) tetralin; $(\square)$ p-xylene; $(\times)$ toluene; $\triangle)$ decalin.

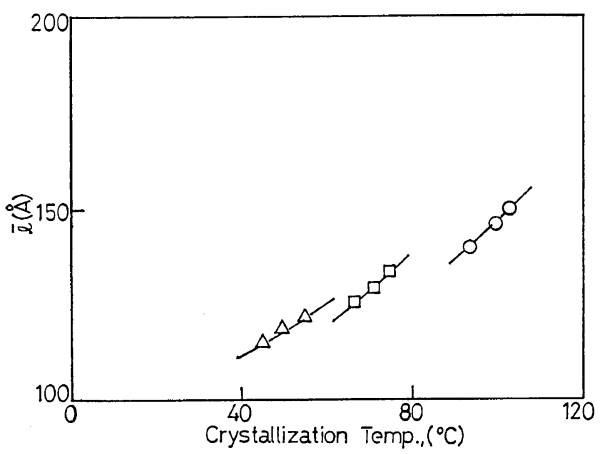

Figure 2. Fold length against crystallization temperature for poly(4-methyl-1-pentene) single crystals grown from dilute solutions: ${ }^{6}(\triangle)$ decalin; $(\square)$ p-xylene; $(\bigcirc)$ tetralin.

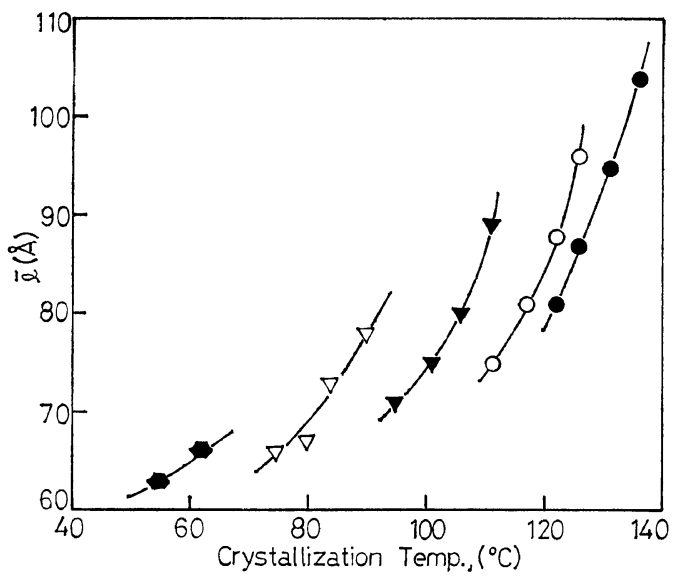

Figure 3. Fold length against crystallization temperature for poly(oxymethylene) single crystals grown from dilute solutions; ${ }^{7}(\circlearrowleft)$ phenol; $(\nabla)$ $m$-cresol; ( $\nabla$ ) furfuryl alcohol; ( $)$ benzyl alcohol; (O) acetophenone.

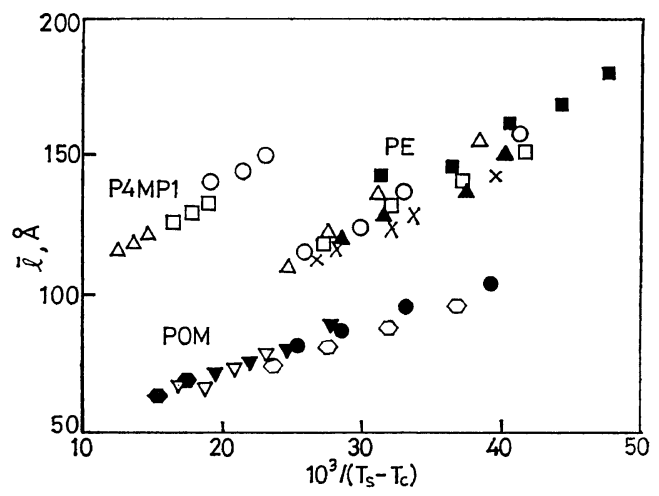

Figure 4. Relation between lamellar thickness $\bar{l}$ and reciprocal of degree of supercooling: $(\triangle)$ decalin; $(\times)$ toluene; $(\bigcirc)$ tetralin; $(\square)$ p-xylene;

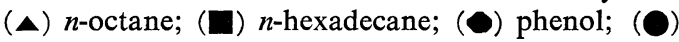
acetophenone; $(\boldsymbol{\nabla}) \mathrm{m}$-cresol; $(\nabla)$ furfuryl alcohol; ( $)$ benzyl alcohol.

are shown in Table I, from previous papers. ${ }^{5-7}$ From these plots, it is seen that the fold length is not only a function of crystallization temperature, but also approximately proportional to the reciprocal of the degree of supercooling. This result strongly supports the kinetic theory. Kinetic theories are based on a specific model envisaging the deposition of molecules by adjacently re-entrant folding occurring in a regularly repeating sequence, while the equilibrium ap- 
Table I. Dissolution temperatures of polyethylene, poly(4-1-methylpentene), and poly(oxymethylene) in various solvents 6,7

\begin{tabular}{|c|c|c|c|c|c|}
\hline \multicolumn{2}{|c|}{ Polyethylene ${ }^{7}$} & \multicolumn{2}{|c|}{ Poly(4-methyl-1-pentene) ${ }^{6}$} & \multicolumn{2}{|c|}{ Poly(oxymethylene) ${ }^{7}$} \\
\hline Solvent & $T_{\mathrm{s}},{ }^{\circ} \mathrm{C}$ & Solvent & $T_{\mathrm{s}},{ }^{\circ} \mathrm{C}$ & Solvent & $T_{\mathrm{s}},{ }^{\circ} \mathrm{C}$ \\
\hline Decalin & 111.3 & Decalin & 123.0 & $m$-Cresol & 132.7 \\
\hline Toluene & 112.5 & Xylene & 127.5 & Furfuryl alc. & 146.5 \\
\hline Xylene & 114.2 & Tetralin & 145.8 & Benzyl alc. & 153.2 \\
\hline Tetralin & 115.1 & & & Acetophenone & 161.3 \\
\hline$n$-Octane & 121.9 & & & & \\
\hline$n$-Hexadecane & 127.8 & & & & \\
\hline
\end{tabular}

proach does not rely on regular folding. Accordingly, the result obtained above may support adjacent re-entrant regular folding as the fold structure of solution-grown single crystals.

\section{Effect of Molecular Weight}

The molecular weight dependence of the fold length was first studied by Keller, et al. $;^{15}$ they determined the fold lengths of solution-grown polyethylene single crystals obtained with various molecular weight fractions and found that the fold length is independent of the length of the polymer molecules, except for the lowest fractions. Kawai, et al.,${ }^{16}$ found that the fold length of the single crystal increases with decreasing molecular weight of the sample, especially in the range of the lower molecular weights, and discussed this result from the viewpoint of kinetic theory. Recently, Sanchez and DiMarzio also discussed the molecular weight dependence of the fold length of solution-grown polyethylene single crystals on the basis of kinetic theory. ${ }^{12}$

The dissolution temperature $T_{\mathrm{s}}$ in eq 3 is defined as that temperature at which the polymer solution is in equilibrium with the extended crystal of that polymer. It increases with increasing molecular weight. But $T_{\mathrm{s}}$ values of polymers having a higher molecular weight than ca. $10^{5}$ are nearly the same as those of crystals of infinitely large size. Therefore, it is expected from eq 3 that the fold length of a single crystal increases with decreasing polymer molecular weight. The dissolution temperature $T_{\mathrm{s}(x)}$ of an extended chain crystal formed with polymers having a degree of polymerization $x$ at a concentration of volume fraction $v_{2}$ is given by: ${ }^{17,18}$

$$
\begin{aligned}
\left(1 / T_{\mathrm{s}(x)}-1 / T_{\mathrm{m}(x)}\right)= & \left(R / \Delta H_{\mathrm{u}}\right)\left\{\left(V_{\mathrm{u}} / V_{1}\right) v_{1}-v_{1} / x\right. \\
& \left.-1 / x \cdot \ln v_{2}-\left(V_{\mathrm{u}} / V_{1}\right) x_{1} v_{1}^{2}\right\}
\end{aligned}
$$

where $T_{\mathrm{m}(x)}$ is the melting temperature of an extended chain crystal having a degree of polymerization $x, R$ is the gas constant, and $v_{1}$ and $v_{2}$ are the volume fractions of the diluent and polymer, respectively, $V_{\mathrm{u}}$ and $V_{1}$ are the molar volumes of the polymer repeating units and the diluents, respectively, and $x_{1}$ is the interaction parameters. From eq 3 and 4, the values of the fold lengths of polyethylene single crystals formed with various molecular weights grown from xylene solution are calculated, assuming appropriate values for $\sigma_{\mathrm{e}}$ and $\delta l$. The input data in eq 3 and 4 are: $\Delta H_{\mathrm{u}}=1960 \mathrm{cal} / \mathrm{mol}$, $\Delta h_{\mathrm{f}}=2.9 \times 10^{9} \mathrm{erg} / \mathrm{cm}^{3}, V_{1}=134.8 \mathrm{ml}, V_{\mathrm{u}}=34.6 \mathrm{ml}$, $x_{1}=0.38,{ }^{5} \quad R=1.987 \mathrm{cal} / \mathrm{mol}, \sigma_{\mathrm{e}}=88 \mathrm{erg} / \mathrm{cm}^{2} .^{7} \delta l$ is assumed to be $46 \AA$ and $58 \AA$ at $T_{\mathrm{c}}=85^{\circ} \mathrm{C}$ and $T_{\mathrm{c}}=70^{\circ} \mathrm{C}$, respectively. $T_{\mathrm{m}(x)}$ is estimated by using the following relation for the melting temperature of the $n$-paraffins, proposed by Broadhurst: ${ }^{19}$

$$
T_{\mathrm{m}(x)}=T_{0} \frac{n+a}{n+b}
$$

where $n$ is the number of carbon atoms per molecule. Constants $a$ and $b$ were found to be $a=-1.5, b=5.0$, and $T_{0}=\lim _{n \rightarrow \infty} T_{\mathrm{m}(n)}=414.3^{\circ} \mathrm{K}$ $\left(141.1^{\circ} \mathrm{C}\right)$.

In Figure 5, the plot of the calculated values of fold length against the molecular weight of polyethylene is shown, along with experimental values. Here the fold length increases with decreasing molecular weight. At higher crystallization temperatures, the dependence of fold length on molecular weight of polyethylene is larger. This result gives further support for the kinetic theory. In this discussion, invariance of the surface free energy with the molecular weight of the sample is assumed. If $\sigma_{\mathrm{e}}$ increases 


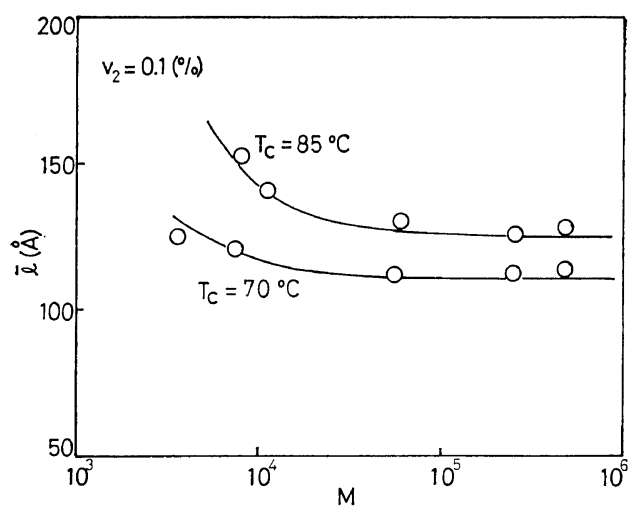

Figure 5. Fold length of polyethylene single crystal grown from xylene solution against molecular weight of solute. Solid lines were calculated from eq 3 and 4 .

with decreasing molecular weight, an alternative explanation for the increase of fold length with decreasing molecular weight would be possible.

\section{Effect of Concentration}

The concentration dependence of the fold length was studied by Basset and $\mathrm{Keller}^{20}$ and Kawai, et al., ${ }^{16}$ who reported that the fold length of solution-grown polyethylene single crystals does not depend on the concentration of the solution in which the crystals are formed, at least up to nearly $1 \%$. From eq 4, it is expected that the dissolution temperature of a single crystal of polymer having a degree of polymerization $x$ may be slightly dependent on the solution concentration. This effect of concentration is very small for single crystals formed with polymers having higher molecular weights than ca. $10^{5}$ and the concentration dependence of the fold length is negligible. But for the single crystal of very much lower molecular weight, the concentration dependence of the fold length is appreciable and the fold length of a single crystal decreases with increasing concentration of solute. From eq 3 and 4, the values of fold lengths of single crystals formed with two polyethylene fractions $\left(M W=7.3 \times 10^{3}\right.$ and $M W=$ $2.59 \times 10^{5}$ ) from xylene solutions of various concentrations can be calculated against the concentration of solute. As input data, the same data as in the preceding section were used, with $\delta l=46 \AA$. In Figure 6 , the plots of the calculated values of fold length against the polymer con-

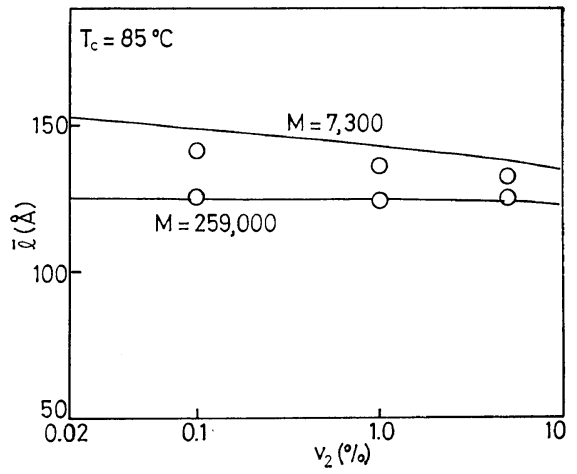

Figure 6. Fold length of polyethylene single crystal grown from xylene solution against concentration of solute. Solid lines were calculated from eq 3 and 4.

centration are shown, along with experimental data. The fold length of a single crystal formed with a higher molecular weight sample $(M W=$ $2.59 \times 10^{5}$ ) shows very little change with respect to polymer concentration, while the fold length of a single crystal formed with a lower molecular weight sample $\left(M W=7.3 \times 10^{3}\right)$ decreases with increasing polymer concentration. The concentration dependence of the fold length of a single crystal is attributed to the concentration dependence of the dissolution temperature predicted from eq 4. This also supports the considerations of kinetic theory.

Now we have to mention the inaccuracies in the determination of $T_{\mathrm{m}(x)}$ and $T_{\mathrm{s}}$. Let us discuss these problems for the case of polyethylene. $T_{\mathrm{m}(x)}$ in eq 4 is estimated by using eq 5 , derived by Broadhurst. ${ }^{19}$ The melting temperature of the extended crystal of polyethylene of infinitely large size is evaluated as $141.1^{\circ} \mathrm{C}$, according to Broadhurst's relation. This value agrees with the melting temperature of an extended crystal of polymethylene crystallized under high pressure. ${ }^{9}$ According to a new relation proposed by Broadhurst, ${ }^{21}$ the melting temperature of an extended crystal of polyethylene of infinitely large size converges to $144.7^{\circ} \mathrm{C}$ and agrees with the melting temperature $145.5^{\circ} \mathrm{C}$ of an extended crystal of polyethylene proposed by Flory and Vrij. ${ }^{22}$ At the present stage, we don't know which temperature is correct $141.1^{\circ} \mathrm{C}$ or $145.5^{\circ} \mathrm{C}$, for the melting temperature of a polyethylene crystal of infinitely 
large size. But even if we adopt the new equation of Broadhurst for $T_{\mathrm{m}(x)}$, calculated value of the fold length has the same tendency in Figures 5 and 6 . The dissolution temperature $T_{\mathrm{s}}$ in eq 2 was obtained by the use of an extended crystal of polymethylene crystallized under high pressure ${ }^{9}$ and the dissolution temperature $T_{\mathrm{s}}$ used by us is lower than the value used by Mandelkern, et al.,${ }^{23}$ which corresponds to $\lim _{n \rightarrow \infty} T_{\mathrm{m}(n)}=145.5^{\circ} \mathrm{C}^{21,22} \quad$ But even if we use the $T_{\mathrm{s}}$ used by Mandelkern, we obtain the same results as obtained for the solvent effect.

Thus all the results obtained above are favorable to a kinetic theory and one may conclude that chain folded polymer crystallization, therefore, normally proceeds by means of the kinetic mechanism of secondary nucleation, although there is a discrepancy between the theoretical and the observed values of the "extra" size term $\delta l$ in eq $3 .^{24,25}$

\section{REFERENCES}

1. A. Peterlin, E. W. Fischer, and C. Reinhold, J. Chem. Phys., 37, 1403 (1962).

2. F. P. Price, J. Chem. Phys., 35, 1884 (1961).

3. J. I. Lauritzen, Jr., and J. D. Hoffman, J. Res. Nat. Bur. Stand., A, 64, 73 (1960).

4. F. C. Frank and M. Tosi, Proc. Roy. Soc., Ser. $A$, 263, 323 (1961).

5. A. Nakajima, E. Hamada, S. Hayashi, and T. Sumida, Kolloid-Z. Z. Polym. 222, 10 (1968).

6. A. Nakajima, S. Hayashi, T. Taka, and N. Utumi, ibid., 234, 1097 (1969).

7. T. Korenaga, F. Hamada, and A. Nakajima,
Polymer J., 321 (1972).

8. S. Hayashi, F. Hamada, A. Saijo, and A. Nakajima, Kobunshi Kagaku (Chem. High Polymers), 24, 769 (1967).

9. B. Wunderlich and T. Arakawa, J. Polym. Sci., Part A, 2, 3967 (1964).

10. K. Hayashi, M. Nishii, and S. Okamura, ibid., Part C, 4, 839 (1963).

11. F. Gornick and J. D. Hoffman, Ind. Eng. Chem., 58, 41 (1966).

12. I. C. Sanchez and E. A. DiMarzio, Macromolecules, 4, 677 (1971).

13. E. W. Fischer and R. Lorenz, Kolloid-Z. $Z$. Polym. 189, 97 (1963).

14. T. Kawai and A. Keller, Phil. Mag., 8, 1208 (1963).

15. A. Keller and A. O'Connor, Polymer (London), 1, 163 (1960).

16. T. Kawai, T. Hama, and K. Ehara, Makromol. Chem., 113, 282 (1968).

17. P. J. Flory, J. Chem. Phys., 17, 223 (1949).

18. A. Nakajima and F. Hamada, Mem. Fac. Eng., Kyoto University, 27, 231 (1965).

19. M. G. Broadhurst, J. Chem. Phys. 36, 2578 (1962).

20. D. C. Bassett and A. Keller, Phil. Mag., 6, 344 (1961).

21. M. G. Broadhurst, J. Res. Nat. Bur. Stand., A, 70, 481 (1966).

22. P. J. Flory and A. Vrij, J. Amer. Chem. Soc., 85, 3548 (1963).

23. J. F. Jackson and L. Mandelkern, Macromolecules, 1, 564 (1968).

24. R. L. Miller, Kolloid-Z. Z. Polym. 225, 62 (1968).

25. D. H. Jones, A. J. Latham, A. Keller, and M. Girolano, J. Polym. Sci., Part A-2, 11, 1759 (1973). 